\title{
Parametrized Stochastic Multi-armed Bandits with Binary Rewards
}

\author{
Chong Jiang and R. Srikant \\ Coordinated Science Laboratory and \\ Dept of Electrical and Computer Engineering \\ University of Illinois at Urbana-Champaign \\ Email: \{jiang17,rsrikant $\} @$ illinois.edu
}

\begin{abstract}
In this paper, we consider the problem of multiarmed bandits with a large, possibly infinite number of correlated arms. We assume that the arms have Bernoulli distributed rewards, independent across time, where the probabilities of success are parametrized by known attribute vectors for each arm, as well as an unknown preference vector, each of dimension $n$. For this model, we seek an algorithm with a total regret that is sub-linear in time and independent of the number of arms. We present such an algorithm, which we call the TwoPhase Algorithm, and analyze its performance. We show upper bounds on the total regret which applies uniformly in time, for both the finite and infinite arm cases. The asymptotics of the finite arm bound show that for any $f \in \omega(\log (T))$, the total regret can be made to be $O(n \cdot f(T))$. In the infinite arm case, the total regret is $O\left(\sqrt{n^{3} T}\right)$.
\end{abstract}

\section{INTRODUCTION}

\section{A. Motivation}

The stochastic multi-armed bandit problem is the following: suppose we are allowed to choose to "pull," or play, any one of $m$ slot machines (also known as one-armed bandits) in each of $T$ timesteps, where each slot machine generates a reward according to its own distribution which is unknown to us. The parameters of the reward distributions are correlated between machines, but the rewards themselves are independent across machines, and independent and identically distributed across timesteps. The choice of which arm to pull may be a function of the sequence of past pulls and the sequence of past rewards. If our goal is to maximize the total reward obtained, taking expectation over the randomness of the outcomes, ideally we would pull the arm with the largest mean at every timestep. However, we do not know in advance which arm has the largest mean, so a certain amount of exploration is required. Too much exploration, though, wastes time that could be spent reaping the reward offered by the best arm. This exemplifies the fundamental trade-off between exploration and exploitation present in a wide class of online machine learning problems.

We consider a model for multi-armed bandit problems in which a large number of arms are present, where the expected rewards of the arms are coupled through an unknown parameter of lower dimension. Now, it is no longer necessary for each arm to be investigated in order to estimate the expected reward from that arm. Instead, we can estimate the underlying parameter; in this way, each pull can yield

Research supported in part by AFOSR MURI FA 9550-10-1-0573. information about multiple arms. We present a simple algorithm, as well as bounds on the expected total regret as a function of time horizon when using this algorithm. While possibly sub-optimal, these bounds are independent of the number of arms.

This model is applicable to certain e-commerce applications: suppose an online retailer has a large number of related products, and wishes to maximize revenue or profit coming from a certain set of customers. If the preferences of this set of customers are known, the list of items which are displayed can be sorted in descending order of expected revenue or profit. However, we may not know a priori what this preference vector is, so we wish to learn online by sequentially presenting each user with an item, observing whether the user buys the item, and then updating an internal estimate of the preference vector.

As a concrete example, imagine an online camera store, with hundreds of different camera models in stock. However, there are perhaps closer to ten features which people will compare when deciding which, if any, to purchase. There are permanent features of the camera itself, such as megapixel count, brand name, and year of introduction, as well as extrinsic features, such as price, review scores, and item popularity. All of these features might be considered by the customer in order to decide whether or not to buy the camera. If bought, the store gains a profit corresponding to the item. A key distinction of our model, when compared to previous work, is the incorporation of this inherently binary choice customers are faced with: to buy or not to buy.

\section{B. Model}

Our model consists of a multi-armed bandit with a set $U$ consisting of $m$ arms (items) and $n$ underlying parameters (attributes), where $m \geq n$ and potentially $m \gg n$. We will interchangeably also think of $U$ as being a $n \times m$ matrix, where each arm $u$ is an $n$-dimensional attribute vector, and is one of the columns of $U$. Furthermore, we will assume that $\operatorname{rank}(U)=n$. There is also a constant but unknown preference vector $z^{*} \in \mathbb{R}^{n}$. The quality $\beta_{u}=u^{T} z^{*}$ of arm $u$ is a scalar indicating how desirable the item is to a user. We will use the logistic function $f$ to define the expected reward of an arm $u$, assuming a particular $z$, as

$$
\alpha_{u}(z)=f\left(u^{T} z\right)=\frac{1}{1+\exp \left(-u^{T} z\right)} .
$$


Thus, the expected rewards of all of the arms are coupled through $z^{*}$. For notational simplicity, we define $\alpha_{u}^{*}=\alpha_{u}\left(z^{*}\right)$. Let the set of equally best arms be

$$
V=\left\{v \in U: \alpha_{v}^{*}=\max _{u \in U} \alpha_{u}^{*}\right\} \subset U .
$$

Define the expected reward of a best arm to be

$$
\alpha_{V}^{*}=\max _{u \in U} \alpha_{u}^{*} \text {. }
$$

At each timestep $t$ up to a finite time horizon $T$, a policy will choose to pull exactly one arm, call this arm $C_{t}$, and a reward $X_{t}$ will be obtained, where $X_{t} \sim \operatorname{Ber}\left(\alpha_{C_{t}}^{*}\right)$. We wish to find policies $g$ which maximize the total expected reward, $\sum_{t=1}^{T} X_{t}$, or equivalently, minimize the expected total regret, $E_{g}\left[\sum_{t=1}^{T}\left(\alpha_{V}^{*}-X_{t}\right)\right]=T \cdot \alpha_{V}^{*}-E_{g}\left[\sum_{t=1}^{T} \alpha_{C_{t}}^{*}\right]$.

\section{Prior Work}

For an introduction and survey of classical multi-armed bandit problems and their variations, see Mahajan and Teneketzis [1]. One of the earliest breakthroughs on the classical multi-armed bandit problem came from Gittins and Jones [2], who showed that under geometric discounting, the optimal policy assigns an index to each arm, now known as the Gittins index, and pulls the arm with the largest Gittins index. Other proofs of this optimality have been given later by Weber [3] and Tsitsiklis [4]. Whittle [5] proved that a similar index-based result is nearly optimal in the "restless bandit" variation of this model, where the arms which are not pulled also evolve in time. While these policies greatly simplify a single $m$-dimensional problem into $m$ 1-dimensional problems, it is still, in general, too computationally complex for online learning.

Lai and Robbins [6] proved an achievable $O(m \cdot \log T)$ lower bound for the expected total regret of the stochastic multi-armed bandit problem in the case of independent arms. Related work by Agrawal et al. [7], [8], [9], [10] and Anantharam et al. [11], [12] considered similar models with i.i.d. and Markov time dependencies for each arm, constructed index policies which are computationally much simpler, and extended the results to include "multiple plays" and "switching costs".

Abe et al. [13] and Auer [14] considered models with finite numbers of arms, with reward distributions that are correlated through a multi-variate parameter $z$ of dimension $n$, and obtained upper bounds on the regret of order $O(\sqrt{m T})$ and $O(\sqrt{n T} \cdot \log T)$, respectively. Mersereau et al. [15] considered a model in which the expected rewards are affine functions of a scalar parameter $z$, but allowed the set of arms to be a bounded, convex region in $\mathbb{R}^{n}$, in which case $m$ is uncountably infinite. They then derived a policy whose expected total regret is $\Theta(\sqrt{T})$. Rusmevichientong and Tsitsiklis [16] expanded this model to allow for a multivariate parameter $z$ of dimension $n$, and showed that the expected total regret (ignoring $\log T$ factors) is $\Theta(n \sqrt{T})$. Dani et al. [17] independently considered a nearly identical model, and obtained similar results. Kleinberg et al. [18] considered a model in which the deterministic rewards are a
Lipschitz-continuous function of the $n$-dimensional vector corresponding to each arm, and obtain an expected total regret (ignoring $\log T$ factors) of $\Theta\left(T^{\frac{n+1}{n+2}}\right)$.

Auer et al. [19] considered a non-stochastic version of the multi-armed bandit problem, in which the rewards are no longer drawn from an unknown distribution, but can instead be adversarially generated. The resultant total weak regret, calculated by comparison with the single arm which is best over the entire time horizon, is shown to be $O(\sqrt{m T})$. The change from logarithmic to polynomial regret in this model is due to having rewards which are time-dependent and potentially adversarially generated, instead of being drawn from a time-independent distribution.

Audibert et al. [20] considered the problem of best arm identification in a stochastic multi-armed bandit setting, but where the goal is to maximize the probability of determining the best arm at the end of a time horizon, as opposed to the usual goal of minimizing total regret over a time horizon. This model is useful when considering exploration and exploitation as occurring in series, instead of in parallel. The probability of error is shown to be upper bounded by a decaying exponential in $T$.

Auer et al. [21] investigated the finite-time regret of the multi-armed bandit problem, assuming bounded but otherwise arbitrary reward distributions. Using upper confidence bound (UCB) algorithms, where the confidence interval of an arm shrinks as the arm is subjected to more plays, they achieve a logarithmic upper bound on the regret, uniform over time, that scales with the "gaps" between the expected rewards for the arms. One algorithm they propose, UCB2, selects the arm with largest empirical mean plus confidence interval, plays it for a number of timesteps dependent on how often that particular arm has been selected in the past, and repeats this process until the time-horizon is reached. This achieves asymptotically optimal expected total regret, and has the best constant possible.

A common idea used in crafting policies to solve the multi-armed bandit problem is that of the doubling trick [22], [23]. This technique is used to convert a parametrized algorithm which works on a time horizon $T$, along with its corresponding bound, into a non-parametrized algorithm that runs forever, with an upper bound that holds uniformly over time.

\section{TWO-PHASE ALGORITHM}

We first present an algorithmic description of a policy for the multi-armed bandit problem described in Section I.B. This algorithm, which we call the Two-Phase Algorithm, will depend on a scheduling function $g: \mathbb{N}_{1} \rightarrow \mathbb{N}_{0}$, such that $g$ is strictly increasing. Since $g$ is not surjective in general, its inverse $g^{-1}$ is not defined over all of $\mathbb{N}_{0}$; however, we can extend the inverse image in the natural way to preserve monotonicity, by defining $g^{-1}: \mathbb{N}_{0} \rightarrow \mathbb{N}_{1}$,

$$
g^{-1}(t)=\max \left\{1 \cup\left\{l \in \mathbb{N}_{1}: g(l) \leq t\right\}\right\} .
$$

In Theorem 3.5. we will show an upper-bound to the expected total regret of this policy on finite arms, which is 
independent of the number of arms $m$. In Theorem 4.6, we will show an upper-bound to the expected total regret of this policy on a special case when there are uncountably infinite arms.

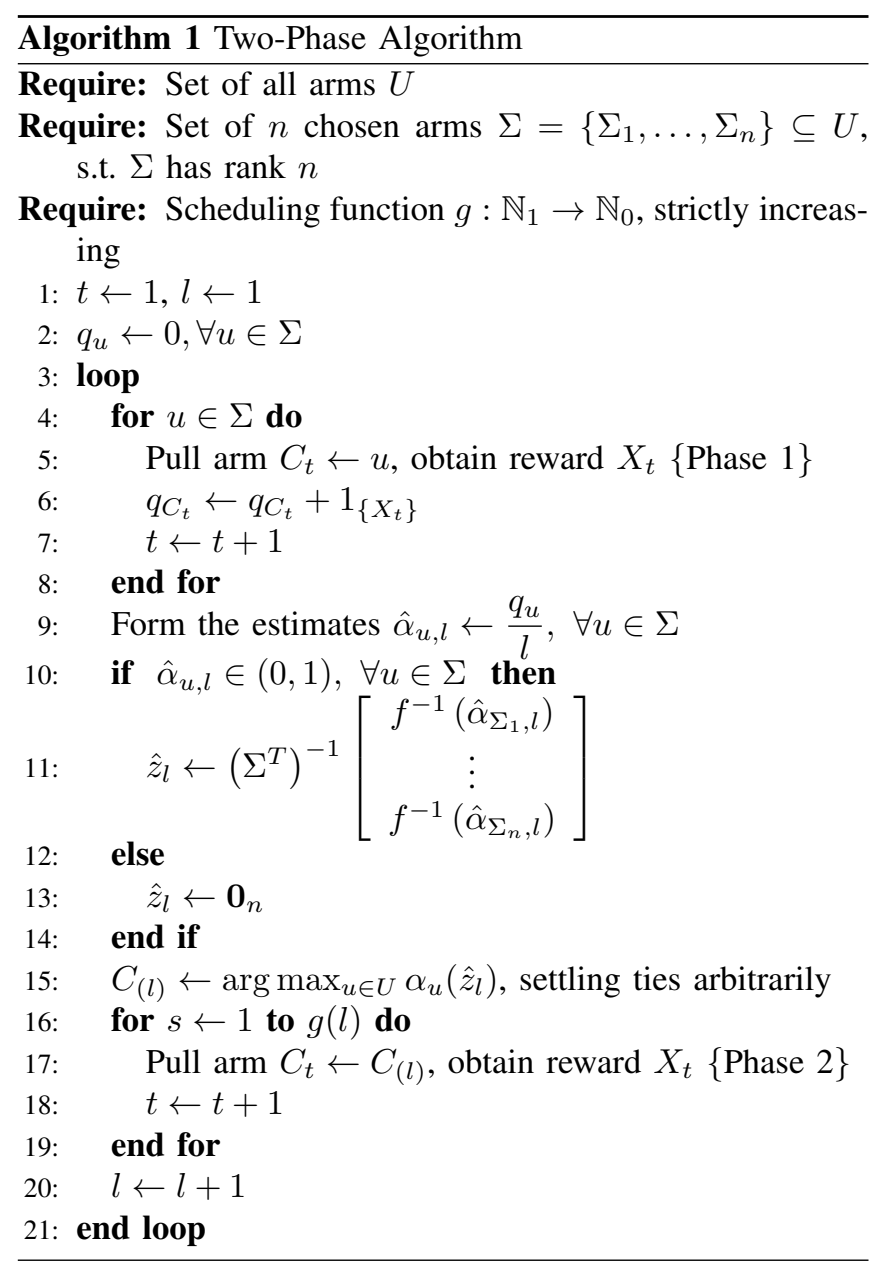

The algorithm requires a selection of $n$ arms,

$$
\Sigma=\left\{\Sigma_{1}, \ldots, \Sigma_{n}\right\} \subseteq U, \text { s.t. } \Sigma \text { has rank } n .
$$

Such a choice exists since we assume $U$ has rank $n$. The algorithm proceeds in epochs; epoch $l$ consists of $n$ exploration pulls (called Phase 1), one for each arm in $\Sigma$, and $g(l)$ exploitation pulls (called Phase 2). In other words, Phase 1 refines our estimate of $z^{*}$, and Phase 2 repeatedly pulls the best arm given our current estimate $\hat{z}_{l}$. If we impose a time horizon of $T$, epochs $1,2, \ldots, L$ are appended until the time horizon $T$ has been reached. The two phases are illustrated in Figure 1 .

For each timestep $t$ in Phase 1 , an arm $u \in \Sigma$ is chosen, and the empirical count of successes $q_{u}$ is incremented if $X_{t}=1$. Prior to each Phase 2 timestep during epoch $l$, there have already been $l$ Phase 1 pulls. We can then form empirical estimates for $\alpha_{i}^{*}$ based on the Phase 1 timesteps, namely $\hat{\alpha}_{u, l}=\frac{q_{u}}{l}, \forall u \in \Sigma$. If

$$
\hat{\alpha}_{u, l} \in(0,1), \forall u \in \Sigma,
$$

then we call epoch $l$ a good epoch, and form the current best estimate for $z^{*}$,

$$
\hat{z}_{l}=\left(\Sigma^{T}\right)^{-1}\left[\begin{array}{c}
f^{-1}\left(\hat{\alpha}_{\Sigma_{1}, l}\right) \\
\vdots \\
f^{-1}\left(\hat{\alpha}_{\Sigma_{n}, l}\right)
\end{array}\right],
$$

since $f$ being strictly increasing and continuous implies $f^{-1}$ exists on $(0,1)$, and since $\Sigma$ being an $n \times n$ matrix with full rank implies $\left(\Sigma^{T}\right)^{-1}$ exists. Otherwise, we call epoch $l$ a bad epoch, and let $\hat{z}_{l}=\mathbf{0}_{n}$. Define the event $G_{l}$ to mean that epoch $l$ is a good epoch. Note that $G_{l} \Longrightarrow G_{l+i} \forall i \in \mathbb{N}_{1}$.

Then, choose an arm

$$
C_{(l)}=\arg \max _{u \in U} \alpha_{u}\left(\hat{z}_{l}\right),
$$

settling ties arbitrarily, and pull this arm $g(l)$ times to form the current epoch's Phase 2.

Remark 2.1: In practice, LU decomposition, instead of matrix inversion, can be used to solve for $\hat{z}_{l}$. Also, since $f$ is strictly increasing, the estimated best arm in a good epoch $l$ can be computed as

$$
C_{(l)}=\arg \max _{u \in U}\left(u^{T} \hat{z}_{l}\right) .
$$

We shall point out some of the ideas behind this algorithm. First, the algorithm is defined to run indefinitely; to obtain the total regret for any finite time horizon $T$, we simply terminate the algorithm when timestep $T$ has been reached. This achieves the same outcome as an application of the doubling trick, in that the algorithm is not dependent on a time horizon $T$. Our algorithm is similar to the algorithm UCB2 of [21]. The main difference is that in our exploration phases, the choice of arm exploits the correlation model that we have assumed in our problem. Furthermore, as we will see later, unlike UCB2, the lengths of the exploitation phases are chosen to grow sub-exponentially in the epoch number (e.g., $g(l) \in \exp (o(l))$ for finite arms) in order to obtain a regret bound that grows slightly faster than logarithmically in the time horizon (e.g., $E\left[R_{T}\right] \in \omega(\log (T))$ for finite arms). As we gain more information and are able to estimate $z^{*}$ more accurately, we can spend a greater fraction of timesteps exploiting the arm we think is best; this is achieved by choosing a suitable scheduling function $g$ to control the ratio of the number of exploitation (Phase 2) pulls versus exploration (Phase 1) pulls, as a function of the epoch number $l$.

Note that there is only randomness in the outcomes $\left\{X_{t}\right\}_{t=1}^{T}$, since the Two-Phase Algorithm is deterministic in the selection of the arm $C_{t}$, conditioned on the history. We will use $\omega$ to denote the sample-paths of $\left\{X_{t}\right\}_{t=1}^{T}$. Let $L$ denote the number of epochs (including partial epochs, as the final one may be truncated) up to timestep $T$, which is independent of sample-path $\omega$. While $L$ is actually a function of $T$, we will not write this dependence explicitly.

Define the expected regret in a single Phase 2 timestep in epoch $l$ to be $E\left[r_{2, l}\right]$. Note that this value is the same for every Phase 2 timestep in epoch $l$, and hence is independent of timestep. Define the total regret up to timestep $T$ in 


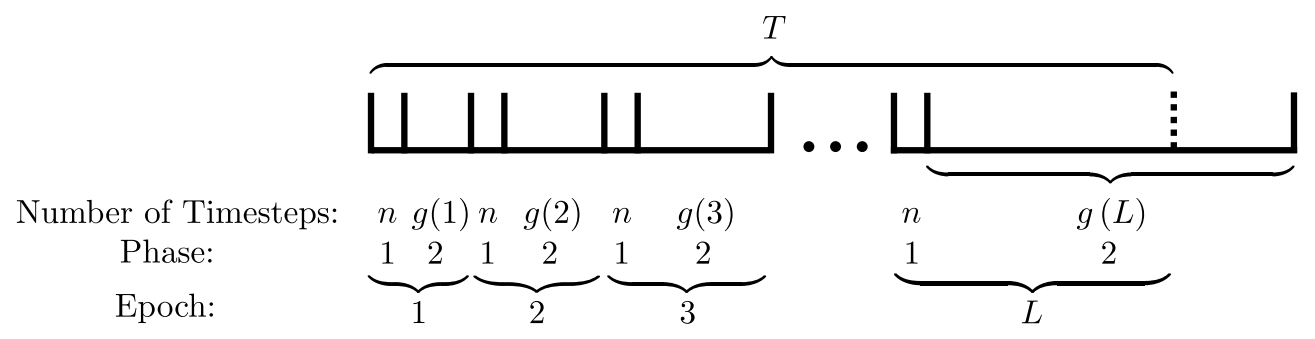

Fig. 1. Given a time horizon $T$, we partition the $T$ timesteps into Phase 1 and Phase 2 timesteps, grouped into a total of $L$ epochs.

the Phase $i$ timesteps for a sample-path $\omega$ to be $R_{i, T}(\omega)$. Define the total regret up to timestep $T$ for a sample-path $\omega$ to be $R_{T}(\omega)=R_{1, T}(\omega)+R_{2, T}(\omega)$. Our goal is to find an upper-bound on $E\left[R_{T}\right]$, the expected total regret. In particular, we are interested in the asymptotic behavior of the upper-bound as $T \rightarrow \infty$.

\section{ANALYSIS, FINITE ARMS}

Consider the multi-armed bandit problem described in Section I.B, where the $m$ arms can each take on any value in $\mathbb{R}^{n}$, as long as $U$ is full rank.

\section{A. Upper-bound Results}

Lemma 3.1: For the Two-Phase Algorithm, we have the following bound on the expected total Phase 1 regret up to timestep $T$ :

Proof:

$$
E\left[R_{1, T}\right] \leq \alpha_{V}^{*} n L
$$

$$
\begin{aligned}
E\left[R_{1, T}\right] & \leq E\left[\sum_{l=1}^{L} \sum_{u \in \Sigma}\left(\alpha_{V}^{*}-\alpha_{u}^{*}\right)\right] \\
& \leq \alpha_{V}^{*} n L .
\end{aligned}
$$

Lemma 3.2: The probability that epoch $l$ is a bad epoch is upper-bounded by

$$
2 n \cdot \exp \left\{-2 l \cdot f\left(-\left\|z^{*}\right\|\right)\right\} .
$$

Proof: In order for epoch $l$ to be a good epoch, we have the condition that $\hat{\alpha}_{u, l} \in(0,1) \forall u \in \Sigma$. Consider the condition for a bad epoch:

$$
\begin{aligned}
& \exists u \in \Sigma \text { s.t. } \hat{\alpha}_{u, l} \notin(0,1) \\
& \Longrightarrow \exists u \in \Sigma \text { s.t. }\left|\hat{\alpha}_{u, l}-\frac{1}{2}\right| \geq \frac{1}{2} \\
& \Longrightarrow \exists u \in \Sigma \text { s.t. }\left|\hat{\alpha}_{u, l}-\alpha_{u}^{*}\right| \geq \frac{1}{2}-\left|\alpha_{u}^{*}-\frac{1}{2}\right| \\
& \Longrightarrow \exists u \in \Sigma \text { s.t. }\left|\hat{\alpha}_{u, l}-\alpha_{u}^{*}\right| \geq \frac{1}{2}-\max _{v \in \Sigma}\left|\alpha_{v}^{*}-\frac{1}{2}\right| .
\end{aligned}
$$

Note that

$$
\max _{v \in \Sigma}\left|\alpha_{v}^{*}-\frac{1}{2}\right| \leq f\left(\max _{u \in \Sigma}\|u\| \cdot\left\|z^{*}\right\|\right)-\frac{1}{2}
$$

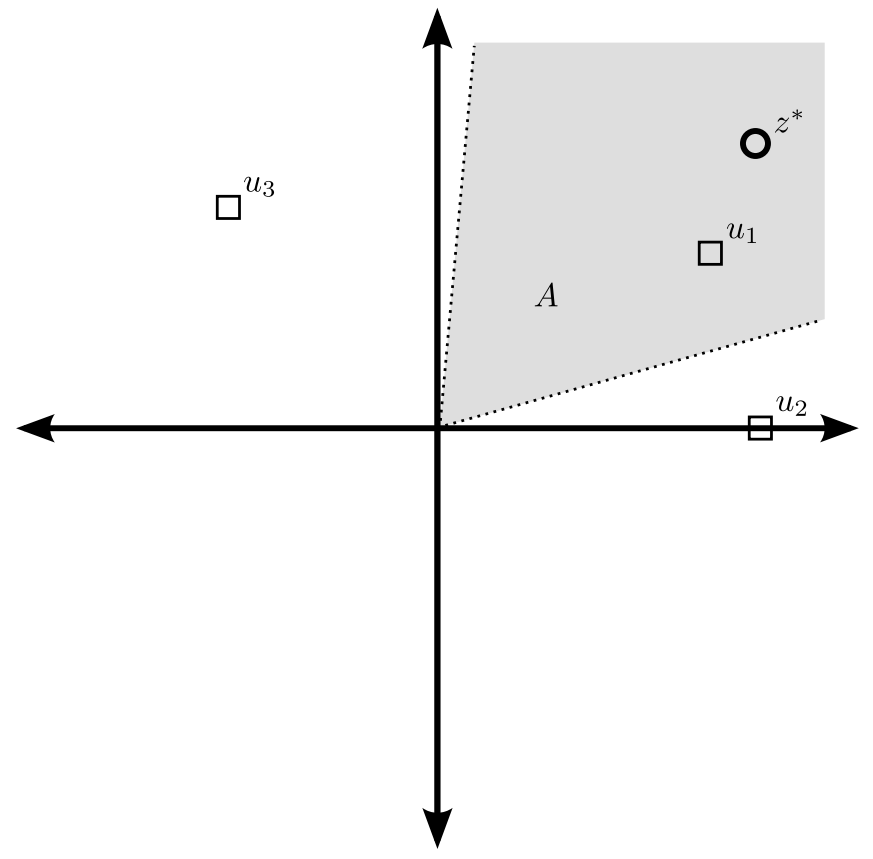

Fig. 2. As an example, consider a scenario with $n=2$ and $m=3$. The arms $U=\left\{u_{1}, u_{2}, u_{3}\right\}$ and the preference vector $z^{*}$ are located at the indicated points. The shaded region is $A$; the boundary of $A$ is formed by the perpindicular bisectors of the segments $u_{3}, u_{1}$ and $u_{1}, u_{2}$.

Then, applying the union bound and Chernoff bound, it follows that

$$
\begin{aligned}
& P\left(\exists i \in \Sigma \text { s.t. } \hat{\alpha}_{i, l} \notin(0,1)\right) \\
\leq & 2 n \cdot \exp \left\{-2 l \cdot\left[1-f\left(\max _{u \in \Sigma}\|u\| \cdot\left\|z^{*}\right\|\right)\right]\right\} \\
= & 2 n \cdot \exp \left\{-2 l \cdot f\left(-\max _{u \in \Sigma}\|u\| \cdot\left\|z^{*}\right\|\right)\right\} .
\end{aligned}
$$

Let $k_{1}=2 f\left(\max _{u \in \Sigma}\|u\| \cdot\left\|z^{*}\right\|\right)$. Thus, the probability that epoch $l$ is a bad epoch is then upper-bounded by

$$
2 n \cdot \exp \left(-k_{1} l\right)
$$

Lemma 3.3: For the Two-Phase Algorithm on finite arms, for a given choice of scheduling function

$$
g \text { s.t. } g(l) \in \exp (o(l))
$$


we have the following bound on the expected Phase 2 regret per timestep in a good epoch $l$ :

$$
E\left[r_{2, l} \mid G_{l}\right] \leq 2 \alpha_{V}^{*} n \cdot \exp (-\gamma l),
$$

where $\gamma$ is a constant which depends on $U$ and $z^{*}$.

Proof: Recall that $\alpha_{u}^{*}=f\left(u^{T} z^{*}\right)$, where

$$
f(\beta)=\frac{1}{1+\exp (-\beta)}
$$

is strictly increasing and continuous. Thus $f^{-1}$ is well defined, strictly increasing and continuous. Recall that

$$
V=\left\{v \in U: \alpha_{v}^{*}=\max _{u \in U} \alpha_{u}^{*}\right\}
$$

is the set of equally best arms. Because $f\left(u_{i}^{T} z\right)$ is continuous in $z$ and defined over $\mathbb{R}^{n}$, it follows that there exists a neighborhood of $z^{*}$, denoted $A$, such that

$$
A=\left\{z \in \mathbb{R}^{n}: \arg \max _{u \in U} \alpha_{u}(z) \in V\right\} .
$$

Since $\Sigma$ is full rank, $A$ must contain an open parallelotope centered at $z^{*}$,

$$
B_{z^{*}}(\delta)=\left\{z \in \mathbb{R}^{n}:\left\|\Sigma^{T} z-\Sigma^{T} z^{*}\right\|_{\infty}<\delta\right\},
$$

where $\delta>0$ and is largest possible. An example of the problem parameters and the induced region $A$ is shown in Figure 2 .

Consider any $z \in B_{z^{*}}(\delta)$. By definition,

$$
\left|u^{T} z-u^{T} z^{*}\right|<\delta, \forall u \in \Sigma .
$$

This is equivalent to

$$
\left|f^{-1}\left(\alpha_{u}(z)\right)-f^{-1}\left(\alpha_{u}^{*}\right)\right|<\delta, \forall u \in \Sigma .
$$

Since $f^{-1}$ is continuous, this is equivalent to having a set of constants

$$
\left\{\underline{\alpha}_{u}, \bar{\alpha}_{u}\right\}_{u \in \Sigma} \text { s.t. } \underline{\alpha}_{u}<\alpha_{u}(z)<\bar{\alpha}_{u},
$$

where

$$
\begin{aligned}
& \underline{\alpha}_{u}=f\left(f^{-1}\left(\alpha_{u}^{*}\right)-\delta\right) \text { and } \\
& \bar{\alpha}_{u}=f\left(f^{-1}\left(\alpha_{u}^{*}\right)+\delta\right), \forall u \in \Sigma .
\end{aligned}
$$

For a Phase 2 timestep during a good epoch $l$, the algorithm forms the empirical average rewards

$$
\hat{\alpha}_{u, l} \in(0,1), \forall u \in \Sigma \text {. }
$$

By the discussion above,

$$
\begin{aligned}
& \underline{\alpha}_{u}<\hat{\alpha}_{u, l}<\bar{\alpha}_{u}, \forall u \in \Sigma \\
\Longrightarrow & \hat{z}_{l} \in B_{z^{*}}(\delta) \subseteq A \\
\Longrightarrow & C_{t}=\arg \max _{u \in U}\left\{u^{T} \hat{z}_{l}\right\} \in V
\end{aligned}
$$

and we will have chosen one of the best arms, accumulating zero regret.
Note that during epoch $l, \hat{\alpha}_{u, l}$ is a sum of $l$ i.i.d. $\operatorname{Ber}\left(\alpha_{u}^{*}\right)$ random variables, $\forall u \in \Sigma$. By the Chernoff bound,

$$
\begin{aligned}
& P\left(\hat{\alpha}_{u, l}<\underline{\alpha}_{u} \mid G_{l}\right) \leq \exp \left[-l \cdot D\left(\underline{\alpha}_{u}|| \alpha_{u}^{*}\right)\right], \text { and } \\
& P\left(\hat{\alpha}_{u, l}>\bar{\alpha}_{u} \mid G_{l}\right) \leq \exp \left[-l \cdot D\left(\bar{\alpha}_{u}|| \alpha_{u}^{*}\right)\right], \forall u \in \Sigma,
\end{aligned}
$$

where $D(p \| q)=p \cdot \log \frac{p}{q}+(1-p) \cdot \log \frac{1-p}{1-q}$ is the $\mathrm{K}-\mathrm{L}$ divergence between two Bernoulli distributions.

Let $\gamma=\min _{u \in \Sigma} \min \left\{D\left(\underline{\alpha}_{u} \| \alpha_{u}^{*}\right), D\left(\bar{\alpha}_{u} \| \alpha_{u}^{*}\right)\right\}$. Note that from the definitions of $\underline{\alpha}_{i}$ and $\bar{\alpha}_{i}$, it follows that

$$
\underline{\alpha}_{u}<\alpha_{u}^{*}<\bar{\alpha}_{u}, \forall u \in \Sigma \text {. }
$$

Since $D(p \| q)=0 \Longleftrightarrow p=q$, we have that $\gamma>0$. By the union bound,

$$
P\left(\exists u \in \Sigma: \hat{\alpha}_{u, l} \notin\left(\underline{\alpha}_{u}, \bar{\alpha}_{u}\right) \mid G_{l}\right) \leq 2 n \cdot \exp (-\gamma l) .
$$

Reviewing the chain of implications, we have

$$
\begin{aligned}
& P\left(\hat{z}_{l} \notin A \mid G_{l}\right) \\
\leq & P\left(\hat{z}_{l} \notin B_{z^{*}}(\delta) \mid G_{l}\right) \\
= & P\left(|| \Sigma^{T} \hat{z}_{l}-\Sigma^{T} z^{*} \|_{\infty}>\delta \mid G_{l}\right) \\
= & P\left(\exists u \in \Sigma:\left|u^{T} \hat{z}_{l}-u^{T} z^{*}\right|>\delta \mid G_{l}\right) \\
= & P\left(\exists u \in \Sigma:\left|f^{-1}\left(\hat{\alpha}_{u, l}\right)-f^{-1}\left(\alpha_{u}^{*}\right)\right|>\delta \mid G_{l}\right) \\
= & P\left(\exists u \in \Sigma: \hat{\alpha}_{u, l} \notin\left(\underline{\alpha}_{u}, \bar{\alpha}_{u}\right) \mid G_{l}\right) \\
\leq & 2 n \cdot \exp (-\gamma l) .
\end{aligned}
$$

Then, we have a bound on the expected per-timestep regret $r_{2, l}$ during Phase 2 of epoch $l$ :

$$
\begin{aligned}
E\left[r_{2, l} \mid G_{l}\right]= & E\left[r_{2, l} \mid \hat{z}_{l} \in A, G_{l}\right] \cdot P\left(\hat{z}_{l} \in A \mid G_{l}\right) \\
& +E\left[r_{2, l}\left|\hat{z}_{l} \notin A\right| G_{l}\right] \cdot P\left(\hat{z}_{l} \notin A \mid G_{l}\right) \\
\leq & 0 \cdot P\left(\hat{z}_{l} \in A \mid G_{l}\right)+\alpha_{V}^{*} \cdot P\left(\hat{z}_{l} \notin A \mid G_{l}\right) \\
\leq & 2 \alpha_{V}^{*} n \cdot \exp (-\gamma l) .
\end{aligned}
$$

Lemma 3.4: For the Two-Phase Algorithm on finite arms, for a given choice of scheduling function

$$
g \text { s.t. } g(l) \in \exp (o(l))
$$

we have the following bound on the expected total Phase 2 regret up to timestep $T$ :

$$
E\left[R_{2, T}\right] \leq \alpha_{V}^{*} n\left(2 k_{2}+L\right)
$$

where $k_{2}$ is a constant which depends on $U$ and $z^{*}$. 
Proof: By Lemmas 3.2 and 3.3, we can upper-bound the expected total Phase 2 regret,

$$
\begin{aligned}
& E\left[R_{2, T}\right] \\
\leq & \sum_{l=1}^{L}\left\{\left[P\left(G_{l}\right) \cdot E\left[r_{2, l} \mid G_{l}\right]+P\left(\neg G_{l}\right) \cdot E\left[r_{2, l} \mid \neg G_{l}\right]\right] \cdot g(l)\right\} \\
\leq & \sum_{l=1}^{L}\left\{\left[P\left(G_{l}\right) \cdot 2 \alpha_{V}^{*} n \cdot \exp (-\gamma l)+P\left(\neg G_{l}\right) \cdot \alpha_{V}^{*}\right] \cdot g(l)\right\} \\
\leq & \sum_{l=1}^{L}\left\{\left[2 \alpha_{V}^{*} n \cdot \exp (-\gamma l)+2 \alpha_{V}^{*} n \cdot \exp \left(-k_{1} l\right)\right] \cdot g(l)\right\} \\
\leq & 2 \alpha_{V}^{*} n \sum_{l=1}^{L^{\prime}}\left\{\left[\exp (-\gamma l)+\exp \left(-k_{1} l\right)\right] \cdot g(l)\right\} \\
& +2 \alpha_{V}^{*} n \sum_{l=L^{\prime}+1}^{L} \frac{1}{2} \\
\leq & \alpha_{V}^{*} n\left\{2 \sum_{l=1}^{L^{\prime}}\left\{\left[\exp (-\gamma l)+\exp \left(-k_{1} l\right)\right] \cdot g(l)\right\}+L\right\},
\end{aligned}
$$

where

$$
L^{\prime}=\max \left\{l:\left[\exp (-\gamma l)+\exp \left(-k_{1} l\right)\right] \cdot g(l)>\frac{1}{2}\right\}
$$

is a constant, independent of sample-path, that depends on $U$ and $z^{*}$ (and is therefore unknown to the algorithm). However, since we have assumed $g(l) \in \exp (o(l))$, it follows that

$$
\lim _{l \rightarrow \infty}\left[\exp (-\gamma l)+\exp \left(-k_{1} l\right)\right] \cdot g(l)=0,
$$

and thus $L^{\prime}$ is finite. Let

$$
k_{2}=\sum_{l=1}^{L^{\prime}}\left\{\left[\exp (-\gamma l)+\exp \left(-k_{1} l\right)\right] \cdot g(l)\right\},
$$

which is well defined since $L^{\prime}$ is finite. Thus,

$$
E\left[R_{2, T}\right] \leq \alpha_{V}^{*} n\left(2 k_{2}+L\right) .
$$

Theorem 3.5: For the Two-Phase Algorithm on finite arms, for a given choice of scheduling function

$$
g \text { s.t. } g(l) \in \exp (o(l)) \text {, }
$$

we have the following bound on the expected total regret up to time-horizon $T$ :

$$
E\left[R_{T}\right] \leq 2 \alpha_{V}^{*} n\left(k_{2}+g^{-1}(T)+1\right) .
$$

Proof: Since the final epoch may be only partially finished, we will lower-bound the total time with the number of timesteps in the penultimate epoch's Phase 2,

$$
T \geq \sum_{l=1}^{L-1}\{n+g(l)\} \geq g(L-1) .
$$

Equivalently,

$$
L \leq g^{-1}(T)+1
$$

Then, using Lemmas 3.1 and 3.4

$$
\begin{aligned}
E\left[R_{T}\right] & =E\left[R_{1, T}\right]+E\left[R_{2, T}\right] \\
& \leq 2 \alpha_{V}^{*} n\left(k_{2}+L\right) \\
& \leq 2 \alpha_{V}^{*} n\left(k_{2}+g^{-1}(T)+1\right) .
\end{aligned}
$$

Corollary 3.6: For the Two-Phase Algorithm on finite arms, for a given choice of scheduling function

$$
g \text { s.t. } g(l) \in \exp (o(l)),
$$

we have the following asymptotic bound on the expected total regret up to time-horizon $T$ :

$$
E\left[R_{T}\right] \in O\left(n \cdot g^{-1}(T)\right) .
$$

Proof: By Theorem 3.5, as a function of $T$,

$$
\begin{gathered}
E\left[R_{T}\right] \leq 2 \alpha_{V}^{*} n\left(k_{2}+g^{-1}(T)+1\right) \\
\in O\left(n \cdot g^{-1}(T)\right),
\end{gathered}
$$

since $\alpha_{V}^{*} \leq 1, k_{2}>0$ is a constant dependent only upon $U$ and $z^{*}$, and $g^{-1}(T) \in \omega(1)$.

\section{Lemma 3.7:}

Proof:

$$
g^{-1}(t) \in \omega(\log (t)) \Longrightarrow g(l) \in \exp (o(l)) .
$$

$$
\begin{aligned}
\lim _{t \rightarrow \infty} \frac{\log (t)}{g^{-1}(t)} & =\lim _{l \rightarrow \infty} \frac{\log (g(l))}{g^{-1}(g(l))} \\
& =\lim _{l \rightarrow \infty} \frac{\log (g(l))}{l} \\
& =0,
\end{aligned}
$$

where (1) is by making the substitution $t=g(l)$, recalling that $g: \mathbb{N}_{1} \rightarrow \mathbb{N}_{0}$ is strictly increasing by assumption, so $\lim _{l \rightarrow \infty} g(l)=\infty$. (2) is since by construction,

$$
g^{-1}(g(l))=l, \forall l \in \mathbb{N}_{1} .
$$

Lastly, (3) is since $g^{-1}(t) \in \omega(\log (t))$, so by definition,

$$
\lim _{t \rightarrow \infty} \frac{g^{-1}(t)}{\log (t)}=\infty
$$

Hence $\log (g(l)) \in o(l)$, so $g(l) \in \exp (o(l))$, and thus $g$ is a valid scheduling function.

Let $\log ^{*}(x)$, the iterated logarithm function, be defined recursively by

$$
\log ^{*}(x)=\left\{\begin{array}{ll}
0, & \text { if } x \leq 1 \\
1+\log ^{*}(\log x), & \text { if } x>1
\end{array} .\right.
$$

Corollary 3.8: The Two-Phase Algorithm can achieve $E\left[R_{T}\right] \in O\left(n \cdot \log (T) \cdot \log ^{*}(T)\right)$.

Proof: Choose

$$
g_{L L S}(l)=\max \left\{t \in \mathbb{N}_{1}: \log (t) \cdot \log ^{*}(t) \leq l\right\} .
$$


Then,

$$
\begin{gathered}
g_{L L S}^{-1}(t)=\left\lfloor\log (t) \cdot \log ^{*}(t)\right\rfloor, \\
\lim _{t \rightarrow \infty} \frac{g_{L L S}^{-1}(t)}{\log (t)}=\lim _{t \rightarrow \infty} \log ^{*}(t) \rightarrow \infty .
\end{gathered}
$$

Thus, $g_{L L S} \in \omega(\log (t))$, and by Lemma 3.7 and Corollary 3.6. we have an achievable expected total regret of

$$
E\left[R_{T}\right] \in O\left(n \cdot g_{L L S}^{-1}(T)\right) \subseteq O\left(n \cdot \log (T) \cdot \log ^{*}(T)\right) .
$$

Remark 3.9: In accordance with other results, such as [6], we suspect this problem has a lower bound that is asymptotically $c n \cdot \log (T)$, where $c$ is dependent on the problem parameters $U$ and $z^{*}$. If this is the case, then by including the term $\log ^{*}(T)$, we are able to obtain an upper bound which is not tight, but within a factor of $\log ^{*}(T)$, while avoiding a dependence on the problem parameters.

\section{B. Generalization to Arm-dependent Rewards}

Suppose that each arm $u \in U$ has a potentially different value of the reward, so that instead of a $\{0,1\}$ reward, it has a $\left\{0, w_{u}\right\}$ reward. Furthermore, suppose that $\left\{w_{u}\right\}_{u \in U}$ is known. Several definitions must be generalized, namely in the model,

$$
\begin{gathered}
X_{t} \sim w_{C_{t}} \cdot \operatorname{Ber}\left(\alpha_{C_{t}}^{*}\right), \\
V=\left\{v \in U: w_{v} \alpha_{v}^{*}=\max _{u \in U} w_{u} \alpha_{u}^{*}\right\} \subset U, \\
w_{V} \alpha_{V}^{*}=\max _{u \in U} w_{u} \alpha_{u}^{*}, \\
A=\left\{z \in \mathbb{R}^{n}: \arg \max _{u \in U} w_{u} \alpha_{u}(z) \in V\right\}, \\
E\left[R_{T}\right]=E_{g}\left[\sum_{t=1}^{T}\left(w_{u} \alpha_{V}^{*}-X_{t}\right)\right],
\end{gathered}
$$

and in the algorithm,

$$
C_{(l)} \leftarrow \arg \max _{u \in U} w_{u} \alpha_{u}\left(\hat{z}_{l}\right) .
$$

Then, Theorem 3.5 generalizes with only minor modifications to the proof, yielding

$$
E\left[R_{T}\right] \leq 2 w_{V} \alpha_{V}^{*} n\left(k_{2}+g^{-1}(T)+1\right) .
$$

Corollaries 3.6 and 3.8 also generalize, with the same results as before.

\section{ANALYSIS, INFINITE ARMS}

Consider the multi-armed bandit problem described in Section I.B, with each point on the unit sphere in $\mathbb{R}^{n}$ being an arm. Now, the number of arms is uncountably infinite, and the finite arm analysis from before no longer yields a useful bound; since there is no longer a gap between the best and second-best arms, the region $A$ degenerates into a line, causing $\gamma=0$ and $k_{2}=\infty$.

\section{A. Upper-bound Results}

For this special case of this infinite arms problem, we shall show that a total expected regret, up to time $T$, of $O\left(\sqrt{n^{3} T}\right)$ is achievable. To obtain a meaningful bound in this case, we eliminate the dependence on $\gamma$, but the trade-off is a worse dependence on $T$. We obtain the $O\left(\sqrt{n^{3} T}\right)$ by analyzing the Two-Phase Algorithm with the choice of arms $\Sigma=\left\{e_{1}, \ldots, e_{n}\right\}$, the standard basis, and the scheduling function $g(l)=\left\lfloor\frac{l}{n}\right\rfloor$.

The proof can be decomposed into several parts.

- First, we have already shown that the probability of an epoch being bad decreases exponentially in the epoch number, in Lemma 3.2 .

- Then, for a good epoch, the probability that $\hat{\alpha}$ deviates from the true value $\alpha^{*}$ also decreases exponentially in the epoch number.

- Large deviations of this estimate can be related to large errors in the central angle between the estimated value $\hat{z}_{l}$ and the true value $z^{*}$.

- Large regret implies large deviations in this central angle.

- Finally, the total expected regret can be bounded using the fact $E\left[r_{2, l}\right]=\int_{0}^{1} P\left(r_{2, l}>\delta\right) d \delta$.

Define $\Theta_{l}$ to be the central angle between $\hat{z}_{l}$ and $z^{*}$ when $\hat{z}_{l} \neq \mathbf{0}_{n}$, and $\pi$ otherwise, i.e.

$$
\Theta_{l}= \begin{cases}\arccos \left(\frac{\left(\hat{z}_{l}\right)^{T} z^{*}}{\left\|\hat{z}_{l}\right\| \cdot\left\|z^{*}\right\|}\right), & \hat{z}_{l} \neq \mathbf{0}_{n} \\ \pi, & \hat{z}_{l}=\mathbf{0}_{n}\end{cases}
$$

Recall that $G_{l}$ denotes the event that epoch $l$ is a good epoch.

Lemma 4.1:

Proof:

$$
r_{2, l}>\delta \Longrightarrow \Theta_{l}>\sqrt{\frac{8 \delta}{\left\|z^{*}\right\|}} .
$$

$$
\begin{aligned}
& r_{2, l}>\delta \\
\Longleftrightarrow & f\left(\left(u^{*}\right)^{T} z^{*}\right)-f\left(\left(\hat{u}_{l}\right)^{T} z^{*}\right)>\delta \\
\Longleftrightarrow & f\left(\frac{\left(z^{*}\right)^{T} z^{*}}{\left\|z^{*}\right\|}\right)-f\left(\left(\hat{u}_{l}\right)^{T} z^{*}\right)>\delta \\
\Longleftrightarrow & f\left(\left\|z^{*}\right\|\right)-f\left(\cos \left(\Theta_{l}\right)\left\|z^{*}\right\|\right)>\delta \\
\Longrightarrow & \left\|z^{*}\right\|\left(1-\cos \left(\Theta_{l}\right)\right)>\frac{\delta}{\min _{x} f^{\prime}(x)}=4 \delta \\
\Longrightarrow & \frac{\Theta_{l}^{2}}{2}>\frac{4 \delta}{\left\|z^{*}\right\|} \\
\Longleftrightarrow & \Theta_{l}>\sqrt{\frac{8 \delta}{\left\|z^{*}\right\|}},
\end{aligned}
$$

where (1) is since $\cos \left(\Theta_{l}\right)=\frac{\left(\hat{z}_{l}\right)^{T} z^{*}}{\left\|\hat{z}_{l}\right\| \cdot\left\|z^{*}\right\|}$, and (2) is since $\cos (x) \geq 1-\frac{x^{2}}{2}, \forall x$. 


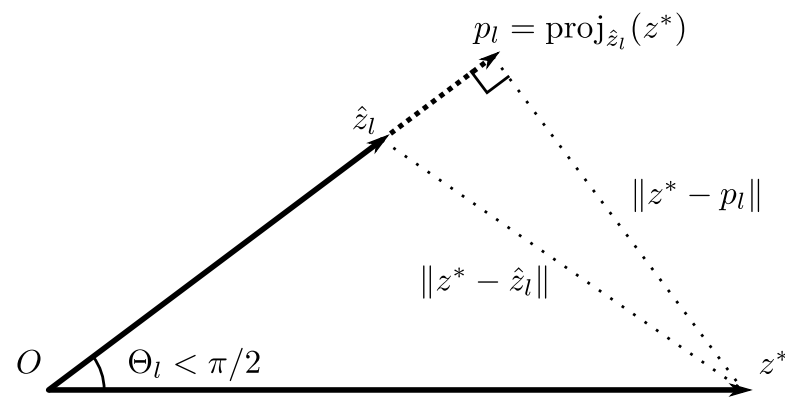

Fig. 3. Schematic diagram illustrating the relationships between $\left\|\hat{z}_{l}-z^{*}\right\|$, $\left\|z^{*}-p_{l}\right\|$, and $\Theta_{l}$.

Lemma 4.2: With the choice $\Sigma=\left\{e_{1}, \ldots, e_{n}\right\}$,

$$
\Theta_{l}>\theta \Longrightarrow \exists u \in \Sigma \text { s.t. }\left|u^{T} \hat{z}_{l}-u^{T} z^{*}\right| \geq \frac{\theta}{\pi \sqrt{n}} \cdot\left\|z^{*}\right\| \text {. }
$$

Proof: Suppose that $\Theta_{l}>\theta$. There are two cases:

$$
\Theta_{l} \in[0, \pi / 2] \text {, or } \Theta_{l} \in(\pi / 2, \pi] .
$$

In the first case, define $p$ to be the vector projection of $z^{*}$ onto $\hat{z}_{l}$; see Figure 3 . Then,

$$
\begin{aligned}
\left\|\hat{z}_{l}-z^{*}\right\| & \geq\left\|z^{*}-p\right\| \\
& =\sin \left(\Theta_{l}\right) \cdot\left\|z^{*}\right\| \\
& >\sin (\theta) \cdot\left\|z^{*}\right\| .
\end{aligned}
$$

In the second case, when $\Theta_{l}>\pi / 2$,

$$
\left\|\hat{z}_{l}-z^{*}\right\| \geq\left\|z^{*}\right\| .
$$

Note that this is true even in the special case of $\hat{z}_{l}=\mathbf{0}_{n}$, by our definition of $\Theta_{l}$. Then, $\forall \theta \in[0,2 \pi]$,

$$
\begin{aligned}
& \Theta_{l}>\theta \\
\Longrightarrow & \left\|\hat{z}_{l}-z^{*}\right\| \geq \frac{\theta}{\pi} \cdot\left\|z^{*}\right\| \\
\Longrightarrow & \exists i \in\{1,2, \ldots, n\} \text { s.t. } \\
& \left|\left(e_{i}\right)^{T} \hat{z}_{l}-\left(e_{i}\right)^{T} z^{*}\right| \geq \frac{\theta}{\pi \sqrt{n}} \cdot\left\|z^{*}\right\| \\
\Longrightarrow & \exists u \in \Sigma \text { s.t. } \\
& \left|u^{T} \hat{z}_{l}-u^{T} z^{*}\right| \geq \frac{\theta}{\pi \sqrt{n}} \cdot\left\|z^{*}\right\| .
\end{aligned}
$$

Lemma 4.3: In a good epoch,

$$
\begin{aligned}
& \exists \tilde{u} \in \Sigma=\left\{e_{1}, e_{2}, \ldots, e_{n}\right\}, \delta_{2} \in(0,1], \text { s.t. } \\
& \left|\tilde{u}^{T} \hat{z}_{l}-\tilde{u}^{T} z^{*}\right| \geq \delta_{2} \cdot\left\|z^{*}\right\| \\
\Longrightarrow & \left|\hat{\alpha}_{\tilde{u}, l}-\alpha_{\tilde{u}}^{*}\right| \geq \delta_{2} \cdot\left\|z^{*}\right\| \cdot f^{\prime}\left(2\left\|z^{*}\right\|\right) .
\end{aligned}
$$

Proof: Suppose the current epoch is good, and

$$
\begin{aligned}
& \exists \tilde{u} \in \Sigma=\left\{e_{1}, e_{2}, \ldots, e_{n}\right\}, \delta_{2} \in(0,1], \text { s.t. } \\
& \left|\tilde{u}^{T} \hat{z}_{l}-\tilde{u}^{T} z^{*}\right| \geq \delta_{2} \cdot\left\|z^{*}\right\| .
\end{aligned}
$$

Since the current epoch is good, $f^{-1}\left(\hat{\alpha}_{u, l}\right)$ exists $\forall u \in \Sigma$. By construction, $\Sigma$ is full rank, and

$$
\hat{z}_{l}=\left(\Sigma^{T}\right)^{-1}\left[\begin{array}{c}
f^{-1}\left(\hat{\alpha}_{\Sigma_{1}, l}\right) \\
\vdots \\
f^{-1}\left(\hat{\alpha}_{\Sigma_{n}, l}\right)
\end{array}\right] .
$$

Thus, $\forall u \in \Sigma$,

$$
\begin{aligned}
& \hat{\alpha}_{u, l}=f\left(u^{T} \hat{z}_{l}\right) \text { and } \\
& \alpha_{u}^{*}=f\left(u^{T} z^{*}\right) .
\end{aligned}
$$

Let $\tilde{\beta}=\tilde{u}^{T} z^{*}+\delta_{2} \cdot\left\|z^{*}\right\| \cdot \operatorname{sgn}\left(\tilde{u}^{T} \hat{z}_{l}-\tilde{u}^{T} z^{*}\right)$. Then,

$$
\begin{aligned}
\left|\hat{\alpha}_{\tilde{u}, l}-\alpha_{\tilde{u}}^{*}\right| & =\left|f\left(\tilde{u}^{T} \hat{z}_{l}\right)-f\left(\tilde{u}^{T} z^{*}\right)\right| \\
& \geq\left|f(\tilde{\beta})-f\left(\tilde{u}^{T} z^{*}\right)\right| \\
& \geq\left|\tilde{\beta}-\tilde{u}^{T} z^{*}\right| \cdot \min _{x \in\left[\tilde{\beta}, \tilde{u}^{T} z^{*}\right]} f^{\prime}(x) \\
& \geq \delta_{2} \cdot\left\|z^{*}\right\| \cdot \min _{x \in\left[-2\left\|z^{*}\right\|, 2\left\|z^{*}\right\|\right]} f^{\prime}(x) \\
& =\delta_{2} \cdot\left\|z^{*}\right\| \cdot f^{\prime}\left(2\left\|z^{*}\right\|\right),
\end{aligned}
$$

where (1) is since $f$ is strictly increasing and

$$
\tilde{\beta} \in\left[\tilde{u}^{T} z^{*}, \tilde{u}^{T} \hat{z}_{l}\right],
$$

(2) is since $f^{\prime}$ is continuous, (3) is since $\delta_{2} \leq 1$ and

$$
\left\|u^{T} z^{*}\right\| \leq\left\|z^{*}\right\| \forall u \in \Sigma,
$$

and thus $\left[\tilde{\beta}, \tilde{u}^{T} z^{*}\right] \subset\left[-2\left\|z^{*}\right\|, 2\left\|z^{*}\right\|\right]$, and (4) is since $f^{\prime}$ is unimodal and symmetric.

\section{Lemma 4.4:}

$$
\begin{aligned}
& P\left(\exists u \in \Sigma:\left|\hat{\alpha}_{u, l}-\alpha_{u}^{*}\right| \geq \delta_{3}\right) \\
\leq & 2 n \cdot \exp \left(-2 l \cdot\left(\delta_{3}\right)^{2}\right) .
\end{aligned}
$$

Proof: Note that $E\left[\hat{\alpha}_{u, l}\right]=\alpha_{u}^{*}, \forall l \in \mathbb{N}_{1}, u \in \Sigma$. Then, for any $u \in \Sigma$, by the Hoeffding bound,

$$
P\left(\left|\hat{\alpha}_{u, l}-\alpha_{u}^{*}\right| \geq \delta_{3}\right) \leq 2 \cdot \exp \left(-2 l \cdot\left(\delta_{3}\right)^{2}\right) .
$$

Applying a union bound over all $u \in \Sigma$ yields the desired result.

\section{Lemma 4.5:}

$$
E\left[r_{2, l} \mid G_{l}\right] \leq \frac{\pi^{2} n^{2}}{8 l \cdot\left\|z^{*}\right\| \cdot f^{\prime}\left(2\left\|z^{*}\right\|\right)^{2}} .
$$


Proof: Combining Lemmas 4.1, 4.2, 4.3 and 4.4, we have that

$$
\begin{aligned}
& P\left(r_{2, l}>\delta \mid G_{l}\right) \\
\leq & P\left(\Theta_{l}>\sqrt{\frac{8 \delta}{\left\|z^{*}\right\|}} \mid G_{l}\right) \\
\leq & P\left(\exists u \in \Sigma:\left|u^{T} \hat{z}_{l}-u^{T} z^{*}\right| \geq \sqrt{\frac{8 \delta}{\pi^{2} n\left\|z^{*}\right\|}} \cdot\left\|z^{*}\right\| \mid G_{l}\right) \\
\leq & P\left(\exists u \in \Sigma:\left|\hat{\alpha}_{u, l}-\alpha_{u}^{*}\right| \geq \sqrt{\frac{8 \delta \cdot\left\|z^{*}\right\|}{\pi^{2} n}} \cdot f^{\prime}\left(2\left\|z^{*}\right\|\right)\right) \\
\leq & 2 n \cdot \exp \left(-l \cdot \frac{16 \delta}{\pi^{2} n} \cdot\left\|z^{*}\right\| \cdot f^{\prime}\left(2\left\|z^{*}\right\|\right)^{2}\right) .
\end{aligned}
$$

Let $k_{3}$ denote the constants independent of $l$ and $n$, namely

$$
k_{3}=\frac{16}{\pi^{2}} \cdot\left\|z^{*}\right\| \cdot f^{\prime}\left(2\left\|z^{*}\right\|\right)^{2} .
$$

Then,

$$
\begin{aligned}
E\left[r_{2, l} \mid G_{l}\right] & =\int_{0}^{1} P\left(r_{2, l}>\delta \mid G_{l}\right) d \delta \\
& \leq \int_{0}^{1} 2 n \cdot \exp \left(-\delta \cdot \frac{k_{3} l}{n}\right) d \delta \\
& =2 n \cdot \frac{-n}{k_{3} l} \cdot\left[\exp \left(-\delta \cdot \frac{k_{3} l}{n}\right)\right]_{0}^{1} \\
& \leq \frac{2 n^{2}}{k_{3} l} .
\end{aligned}
$$

Theorem 4.6: For the Two-Phase Algorithm on a unit sphere of arms in $\mathbb{R}^{n}$, we have the following bound on the total expected regret up to time-horizon $T$ :

$$
E\left[R_{T}\right] \leq\left(1+\frac{2}{k_{3}}\right) \cdot n L+2 \sum_{l=1}^{L} l \cdot \exp \left(-k_{4} l\right) .
$$

Proof: Since the final epoch may be only partially finished, we will lower-bound the total time with the number of timesteps in all prior epochs. Decomposing into Phase 1 and Phase 2, we have

$$
\begin{aligned}
T & \geq \sum_{l=1}^{L-1}\left\{n+\left\lfloor\frac{l}{n}\right\rfloor\right\} \\
& \geq \sum_{l=1}^{L-1} \frac{l}{n} \\
& \geq \frac{(L-1)^{2}}{2 n} .
\end{aligned}
$$

Equivalently,

$$
L \leq \sqrt{2 n T}+1
$$

By Lemmas 3.2 and 4.5, we can upper-bound the expected total Phase 2 regret,

$$
\begin{aligned}
& E\left[R_{2, T}\right] \\
\leq & \sum_{l=1}^{L}\left\{\left[P\left(G_{l}\right) \cdot E\left[r_{2, l} \mid G_{l}\right]+P\left(\neg G_{l}\right) \cdot E\left[r_{2, l} \mid \neg G_{l}\right]\right] \cdot\left\lfloor\frac{l}{n}\right\rfloor\right\} \\
\leq & \sum_{l=1}^{L}\left\{\left[P\left(G_{l}\right) \cdot \frac{2 n^{2}}{k_{3} l}+P\left(\neg G_{l}\right) \cdot 1\right] \cdot \frac{l}{n}\right\} \\
\leq & \sum_{l=1}^{L}\left\{\left[\frac{2 n^{2}}{k_{3} l}+2 n \cdot \exp \left(-k_{1} l\right)\right] \cdot \frac{l}{n}\right\} \\
\leq & \frac{2 n L}{k_{3}}+2 \sum_{l=1}^{\infty} l \cdot \exp \left(-k_{1} l\right) .
\end{aligned}
$$

Then, using Lemma 3.1

$$
\begin{aligned}
E\left[R_{T}\right]= & E\left[R_{1, T}\right]+E\left[R_{2, T}\right] \\
\leq & \left(\alpha_{V}^{*}+\frac{2}{k_{3}}\right) \cdot n L+2 \sum_{l=1}^{\infty} l \cdot \exp \left(-k_{1} l\right) \\
\leq & \left(\alpha_{V}^{*}+\frac{2}{k_{3}}\right) \cdot\left(\sqrt{2 n^{3} T}+n\right) \\
& +2 \sum_{l=1}^{\infty} l \cdot \exp \left(-k_{1} l\right) .
\end{aligned}
$$

Corollary 4.7: For the Two-Phase Algorithm on a unit sphere of arms in $\mathbb{R}^{n}$, we have the following asymptotic bound on the expected total regret up to timestep $T$ :

$$
E\left[R_{T}\right] \in O\left(\sqrt{n^{3} T}\right) .
$$

Proof: By Theorem 4.6, as a function of $T$,

$$
\begin{aligned}
E\left[R_{T}\right]= & \left(\alpha_{V}^{*}+\frac{2}{k_{3}}\right) \cdot\left(\sqrt{2 n^{3} T}+n\right) \\
& +2 \sum_{l=1}^{\infty} l \cdot \exp \left(-k_{1} l\right) \\
\in & O\left(\sqrt{n^{3} T}\right),
\end{aligned}
$$

since $\alpha_{V}^{*} \leq 1$, and $k_{1}, k_{3}>0$ are both constants dependent only upon $\left\|z^{*}\right\|$.

Remark 4.8: The choice of scheduling function $g$ is not restricted to be $\left\lfloor\frac{l}{n}\right\rfloor$; the dependence on $n$ can be altered to change the trade-off between the constants in front of $\sqrt{T}$ and $\sum_{l=1}^{\infty} l \cdot \exp \left(-k_{1} l\right)$. That is, the asymptotics can be improved at the expense of short time-horizon performance. Furthermore, if the time-horizon is known in advance, then the scheduling function can be chosen to minimize the sum of these two terms, just as in the finite arm case.

\section{CONCLUSIONS}

We have proposed a class of parametrized multi-armed bandit problems, in which the reward distribution is Bernoulli and independent across arms and across time, with a parameter that is a non-linear function of the scalar quality of an 
arm. The real-valued qualities are inner products between the unknown preference and known attribute vectors. Under this model, we are able to capture the fundamentally binary choice inherent in certain online machine learning problems.

Our proposed algorithm achieves an asymptotic expected total regret of $O\left(n \cdot g^{-1}(T)\right)$ for any function $g^{-1}(T) \in \omega(\log (T))$ in the finite arm case, and $O\left(\sqrt{n^{3} T}\right)$ in the infinite arm, unit circle case. This is in contrast to the $\Omega(m \log (T))$ lower-bound of Lai and Robbins, and the $\Omega\left(T^{\frac{n+1}{n+2}}\right)$ lower-bound of Kleinberg et al. In both cases, the additional assumption of structure (linearly correlated instead of independent, and logistic function of linear instead of Lipschitz, respectively) can be used to out-perform optimal algorithms which do not account for this structure. We conjecture that the lower-bounds on our problem are $\Omega(n \cdot \log (T))$ and $\Omega(\sqrt{(T)})$ for the finite and infinite arm cases, respectively; if true, then this simple algorithm's performance is nearly optimal.

Finally, our algorithm can be implemented very efficiently, since the storage requirements are $O(n)$ and thus do not scale with either the number of arms or the time-horizon. Also, since the exporation and expoitation phases are decoupled, the only history-dependent part of the algorithm, the optimization to determine which arm to pull, is only performed during a small number of timesteps (approximately $O(\log (T))$ and $O(\sqrt{T})$ for finite and infinite arm cases, respectively). In the infinite arm, unit circle case, this optimization itself is simply the normalization of the current estimate $\hat{z}_{l}$.

The basic idea of increasing the length of epochs is similar to that of UCB2, but because our algorithm uses a global count of the epoch instead of local counts for each arm, it is applicable to infinite arm problems. Finally, we note that several extensions to this work are possible; multiple plays and time-dependent $U$ and $z^{*}$ would be directly applicable for e-commerce applications.

\section{REFERENCES}

[1] A. Mahajan and D. Teneketzis, "Multi-armed bandit problems," in Foundations and Applications of Sensor Management, A. O. Hero, D. A. Castañón, D. Cochran, and K. Kastella, Eds. Springer-Verlag, 2007, ch. 6, pp. 121-151.

[2] J. C. Gittins and D. M. Jones, "A dynamic allocation index for the sequential design of experiments," Progress in Statistics, vol. 1, pp. 241-266, 1974.

[3] R. Weber, "On the Gittins index for multiarmed bandits," The Annals of Applied Probability, vol. 2, no. 4, pp. 1024-1033, Nov. 1992.
[4] J. N. Tsitsiklis, "A short proof of the Gittins index theorem," The Annals of Applied Probability, vol. 4, no. 1, pp. 194-199, Feb. 1994.

[5] P. Whittle, "Restless bandits: Activity allocation in a changing world," Journal of Applied Probability, vol. 25, pp. 287-298, 1988.

[6] T. L. Lai and H. Robbins, "Asymptotically efficient adaptive allocation rules," Advances in Applied Mathematics, vol. 6, pp. 4-22, 1985.

[7] R. Agrawal, D. Teneketzis, and V. Anantharam, "Asymptotically efficient adaptive allocation schemes for controlled i.i.d. processes: Finite parameter space," IEEE Transactions on Automatic Control, vol. 34, no. 3, pp. 258-267, Mar. 1989.

[8] — - "Asymptotically efficient adaptive allocation schemes for controlled Markov chains: Finite parameter space," IEEE Transactions on Automatic Control, vol. 34, no. 12, pp. 1249-1259, Dec. 1989.

[9] R. Agrawal, M. Hegde, and D. Teneketzis, "The multi-armed bandit problem with switching cost," in 26th IEEE Conference on Decision and Control, 1987, vol. 26, Dec. 1987, pp. 1106-1108.

[10] R. Agrawal, "Sample mean based index policies with $o(\log n)$ regret for the multi-armed bandit problem," Advances in Applied Probability, vol. 27, no. 4, pp. 1054-1078.

[11] V. Anantharam, P. Varaiya, and J. Walrand, "Asymptotically efficient allocation rules for the multiarmed bandit problem with multiple plays-Part I: I.I.D. rewards," IEEE Transactions on Automatic Control, vol. 32, no. 11, pp. 968-976, Nov. 1987.

[12] _ - "Asymptotically efficient allocation rules for the multiarmed bandit problem with multiple plays-Part II: Markovian rewards," IEEE Transactions on Automatic Control, vol. 32, no. 11, pp. 977-982, Nov. 1987.

[13] N. Abe, A. W. Biermann, and P. M. Long, "Reinforcement learning with immediate rewards and linear hypotheses," Algorithmica, vol. 37, no. 4, pp. 263-293, 2003.

[14] P. Auer, "Using confidence bounds for exploitation-exploration tradeoffs," Journal of Machine Learning Research, vol. 3, pp. 397-422, March 2003.

[15] A. J. Mersereau, P. Rusmevichientong, and J. N. Tsitsiklis, "A structured multiarmed bandit problem and the greedy policy," IEEE Transactions on Automatic Control, vol. 54, no. 12, pp. 2787-2802, Dec. 2009.

[16] P. Rusmevichientong and J. N. Tsitsiklis, "Linearly parameterized bandits," Mathematics of Operations Research, vol. 35, no. 2, pp. 395411, May 2010.

[17] V. Dani, T. P. Hayes, and S. M. Kakade, "Stochastic linear optimization under bandit feedback," in Proc. of the 21st Annual Conference on Learning Theory, Helsinki, Finland, July 2008, pp. 363-374.

[18] R. Kleinberg, A. Slivkins, and E. Upfal, "Multi-armed bandits in metric spaces," in Proc. of the 40th annual ACM symposium on Theory of computing, Victoria, British Columbia, Canada, 2008, pp. 681-690.

[19] P. Auer, N. Cesa-Bianchi, Y. Freund, and R. E. Schapire, "The nonstochastic multiarmed bandit problem," SIAM Journal on Computing, vol. 32, no. 1, pp. 48-77, 2002.

[20] J.-Y. Audibert, S. Bubeck, and R. Munos, "Best arm identification in multi-armed bandits," in Proc. of the 23rd Annual Conference on Learning Theory, Haifa, Israel, June 2010, pp. 41-53.

[21] P. Auer, N. Cesa-Bianchi, and P. Fischer, "Finite-time analysis of the multiarmed bandit problem," Machine Learning, vol. 47, no. 2, pp. 235-256, 2002.

[22] G. Stoltz, "Incomplete information and internal regret in prediction of individual sequences," Ph.D. dissertation, University of Paris-Sud, Nov. 2005. [Online]. Available: http://eprints.pascal-network.org/ archive/00001692/

[23] N. Cesa-Bianchi and G. Lugosi, Prediction, Learning, and Games. New York, NY: Cambridge University Press, 2006. 